\title{
TRNSYS Simulation of Hybrid Ground Source Heat Pump System Based on Cooling Conditions
}

\author{
Jun Zhang ${ }^{1, a^{*}}$, Shunyu Su ${ }^{1, b^{*}}$, Lamei Liü ${ }^{1, \mathrm{c}}$, Chuanhui Zhou ${ }^{1, \mathrm{~d}}$, Lei Shi ${ }^{2, \mathrm{e}}$ \\ ${ }^{1}$ College of Urban Construction, Wuhan University of Science and Technology, Wuhan, China \\ ${ }^{2}$ Zhejiang Loopmaster Energy Technology Co, Ltd, Hangzhou 310051, China

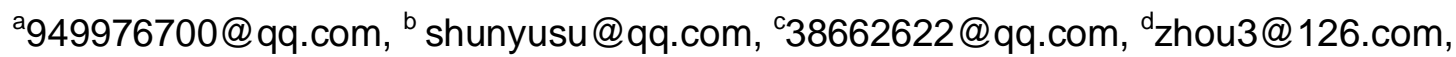 \\ e176785813@qq.com
}

\begin{abstract}
Keywords: Ground source heat pump system; Lake Water source heat pump system; Temperature differential control

Abstract: By importing the meteorological data of water temperatures to TRNSYS software, temperature differential control method was applied to simulate hybrid ground source heat pump system for three months in Wuhan. In order to obtain running effect of this system, the inlet and outlet water temperatures of heat pump and buried pipe, as well as the soil temperature change trend and the COP of this system were investigated respectively. The results show that this system is running well under given conditions and it provides reference for the operation of hybrid ground source heat pump system.
\end{abstract}

\section{Introduction}

The ground source side of the ground source heat pump system includes underground soil, surface water and underground water. This system could transfer the heat in soil to the place requiring heating in winter, and transfer indoor heat out through the electric consumption to achieve cooling goal in summer. So it is an effective way to use energy. Ground source heat pump system is widely used in engineering practice in recent years and made a great contribution to energy conservation and emissions reduction [1].

Ground source heat pump has been widely used in Wuhan, which is in the hot summer and cold winter area. But the heat discharged into the soil in summer is greater than that obtained from the soil in winter. By accumulating year after year, it will lead to temperature rise and reduce heat transfer capacity of the soil, thereby affecting the energy efficiency of ground source heat pump system. This problem should be effectively solved by using soil and surface water mixed operation [2-4]. And the details will be discussed in this paper.

\section{The composition of surface water heat exchange}

Heat exchanges between surface water and its surroundings mainly include solar radiation, sky radiation, evaporation, convective heat transfer to the air, and heat conductivity to the earth. Surface water heat transfer process is illustrated below.

Figure 1 shows that the heat gain of surface water is mainly from the sun radiation. There is about $40 \%$ of the solar radiation heat absorbed by the water surface if the water in lake is clear. And the heat is mainly absorbed by the upper part of water. Surface water cooling is mainly through transferring radiation heat to the surroundings at night. For example, when the temperature difference reaches $14 \mathrm{k}$, the thermal radiation is about $160 \mathrm{~W} / \mathrm{m}^{2}$. 


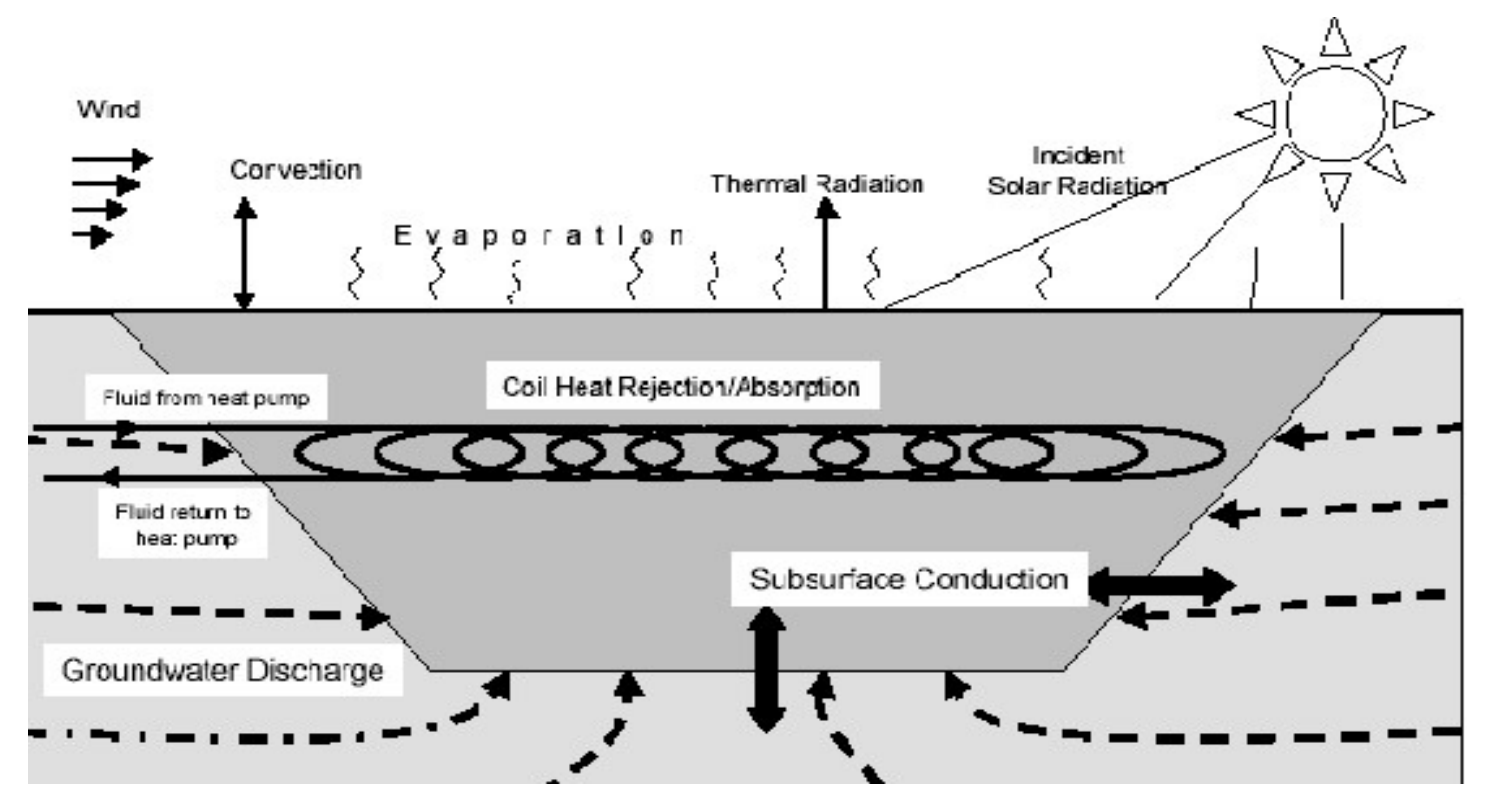

Fig.1. Heat transfer mechanism in a pond

The other method of heat gain is convection heat transfer. This part is very small, and usually only about 10\% 20\% of solar radiation heat gain. When the lake is at a lower temperature, it will get heat by thermal convection. The increase of wind speed will benefit to the convective heat transfer of the lake. Water cooling is also completed through the surface evaporation.

The last part for the heat transfer is heat conduction through the soil. It is only a small part in total heat transfer, and mainly influences bottom temperature of the lake water $[5,6]$.

\section{TRNSYS simulation}

TRNSYS (Transient System Simulation Program) is mainly used for building thermal environmental simulation and analysis. The biggest feature of this software is modular analytical methods. When simulating the air conditioning system, with the methods of set -- calculation -feedback -- set - calculation and through feedback, the user can correct system settings to make the calculation more precise. And all standard modules in TRNSYS software source code are open, so the user can modify and add module when it is necessary [7].

\section{The establishment of model}

The establishment of the shallow water model. When the depth of the water does not exceed $4 \sim 5 \mathrm{~m}$, it can be assumed that the water is mixed well, namely the water temperature is consistent, and the internal temperature gradient is ignored. According to the first law of thermodynamics, the single node heat transfer model is built as following.

$$
q_{\text {in }}-q_{o u t}=V * \rho * C_{p} \frac{d T}{d t}
$$

Where $q_{\mathrm{in}^{-}}{ }^{-- \text {Water absorption heat }}$
$q_{\text {out }}-$ Water release heat 


$$
\begin{aligned}
& \mathrm{V} \text {-- The volume of water } \\
& \mathrm{p}-- \text { Water density } \\
& \mathrm{C}_{\mathrm{p}} \text {-- Specific heat of water } \\
& \frac{\mathrm{dT}}{\mathrm{dt}} \text {-- Water temperature on the derivative of the time }
\end{aligned}
$$

Shallow water bodies mainly obtain heat through solar radiation, heat convection, the earth thermal conductivity and evaporation heat transfer. The heat in shallow water heat exchanger will be increased. The changes of water temperature with time can be expressed as following differential equation.

$$
\frac{d T}{d t}=\frac{q_{\text {solar }}+q_{\text {longwave }}+q_{\text {vomvection }}+q_{\text {graund }}+q_{\text {svaparation }}+q_{\text {fluid }}}{V * p * c_{p}} .
$$

Where q zolar --solar heat

$q_{\text {longuave }}$--Long wave radiation heat

$q_{\text {convection }}$--Convective heat transfer heat

$\mathrm{q}_{\text {ground }}$--The ground conduction heat

$\mathrm{q}_{\text {evaporation }}$--Evaporation transfer heat

$q_{\text {fluid }}$-- Shallow water heat exchanger heat

When surface water body reaches a certain depth, it produces obvious stratified phenomenon. The biggest density of water is $4.0^{\circ} \mathrm{C}$, rather than freezing temperature $0^{\circ} \mathrm{C}$. It causes thermal stratification of surface water [4].

Soil heat exchanger model. Borehole region of underground pipe generally comprises ground pipe, circulating medium in ground pipe, soil backfill material in borehole and soil outside the borehole. The heat transfer formula of buried pipe heat exchanger is built based on the principles of heat transfer. The inner part of the borehole is processed as a steady-state heat transfer model. And the outer part of the borehole is processed as an unsteady heat transfer model [8].

Soil heat exchanger module is expressed with Type 557 in TRNSYS. This module is compiled with DST calculation model, which is able to exchange heat with the heat transfer medium within the soil and give a very accurate analysis calculation.

\section{Simulation analysis of hybrid system}

Operation mode. By importing an office building in Wuhan University of Science and Technology and the water temperature meteorological parameters, the changes of soil temperature in summer from June to August are simulated. The amount of heat discharged into the water should be controlled in order to avoid excessive fluctuations of water temperatures. The soil source heat pump is the main operation system and the surface water source heat pump is auxiliary system in this model.

The temperature control method is applied in this paper [9]. Since the setting temperature difference is smaller, the surface water system will be running more frequently, and the power 
consumption of the surface water side will increase. So the energy consumption of this system increases with the decrease of the temperature difference. Therefore the difference of outdoor environment wet bulb temperature and the heat pump units fluid inlet temperature is supposed to $4^{\circ} \mathrm{C}$. When the temperature difference is more than $4^{\circ} \mathrm{C}$, the system open the surface water side, operating together with the soil source side, and the surface water side absorbs extra quantity of heat. When it is less than $4^{\circ} \mathrm{C}$, the system will only operate in soil source heat pump side.

System diagram. Figure 2 shows the system diagram in TRNSYS.

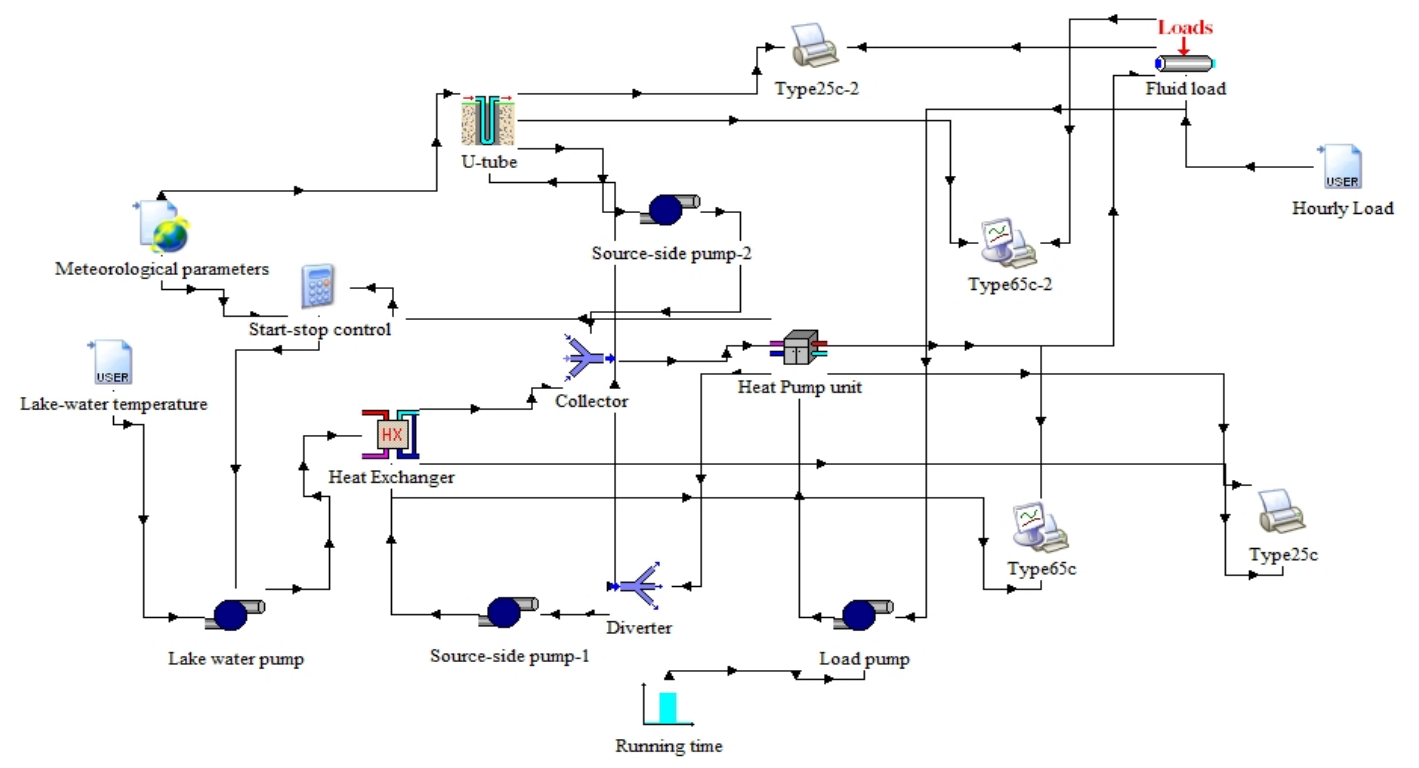

Fig.2. System diagram

\section{Simulation results and analysis}

Figure 3 shows that the highest inlet water temperature of buried pipe is about $14.0^{\circ} \mathrm{C}$, and the corresponding outlet water temperature is about $9.5^{\circ} \mathrm{C}$. The temperature difference is $4.5^{\circ} \mathrm{C}$. The highest inlet water temperature of the heat pump units is about $35.9^{\circ} \mathrm{C}$, and the corresponding outlet water temperature is about $30.1^{\circ} \mathrm{C}$. The temperature difference is $5.8^{\circ} \mathrm{C}$. The water temperature of the heat pump units maintained relatively stable by running for three mouth, but the average ground temperature decreases from $19.8^{\circ} \mathrm{C}$ to $15.4^{\circ} \mathrm{C}$. The temperature drop is $4.4^{\circ} \mathrm{C}$. If the operation of this system continues, the fall of geothermal temperature will also continue. It will benefit to the operation of this system. With the transitional season coming, soil temperature will recover.

Figure 4 shows that the COP of this system is gradually reducing, but the trend is very smooth. The COP is basically above 5.8, and the system maintains a reasonable level of energy consumption ratio. It means that this system is running well. 


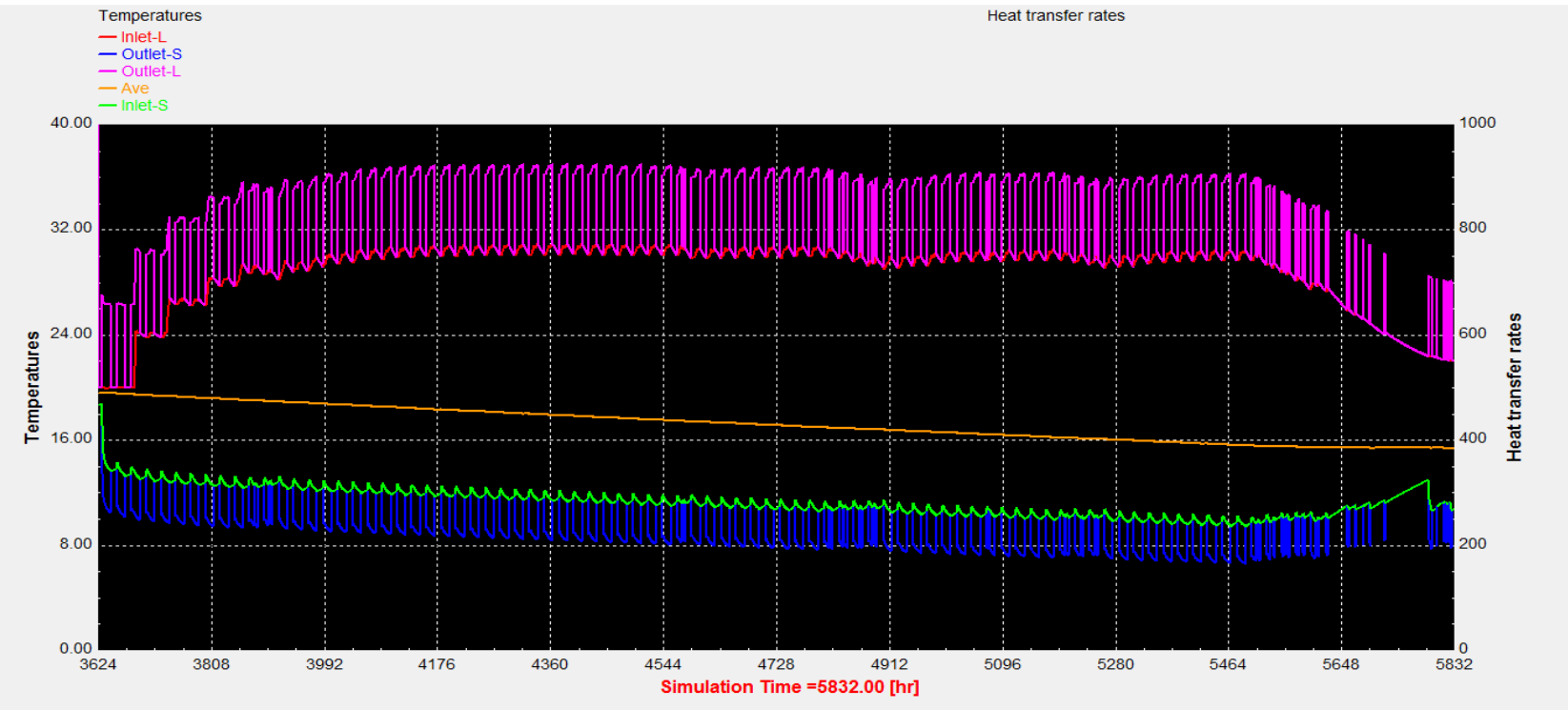

Fig.3. Inlet and outlet water temperatures of buried pipe and heat pump

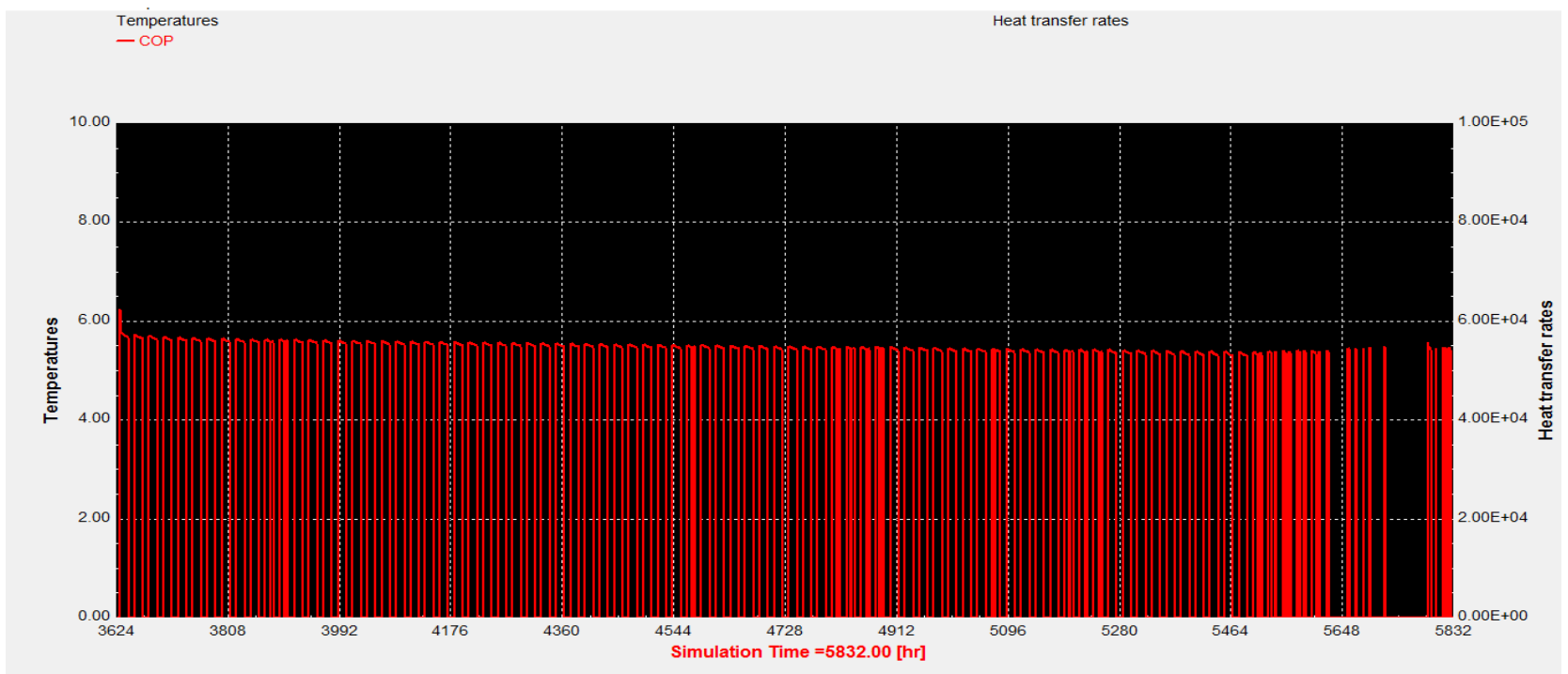

Fig.4. COP of the System

\section{Conclusions}

The combined system of soil source heat pump and lake water source heat pump in Wuhan was simulated with TRNSYS software in this paper. The data of hybrid system running for three months were obtained while the difference between the wet bulb temperature of outdoor ambient and the temperature of heat pump fluid inlet is $4^{\circ} \mathrm{C}$. The analytical results show that the operation of this system is stable, and the soil temperatures in summer gradually decrease as the running time going on while the temperature difference is about $4.4^{\circ} \mathrm{C}$. The temperature drop is significant, but it is not a remarkable decrease. As the transitional season come, soil temperature will be restored. And the system can run well under the mentioned conditions. 


\section{Acknowledgements}

The authors wish to thank the financial support of China Hubei Provincial Natural Science Foundation (No. 2014CFB234)

\section{References}

[1] Hu Pinfang, Huang Zhi, Sun Xinming, Development status of study and application of ground-source heat pump technology, Refrigeration and Air Conditioning, Vol.14,2014

[2] Tim Peer, P.E.; W. \& (Lanny) Joyce, P.E., Lake-Source Cooling. ASHRAE Journal, Vol.44

[3] Kavanagh, S P., Pezent, M.C, Lakewater Applications of Water-to-Air Heat Pumps, ASHRAE Trans, 1990, Vo1. 96.

[4] Bhargava, A, Trabia, M, Moujaes, S F. Optimal Design of a Cold-Sea-Water Chiller. ASHRAE Trans. 1994, Vol 100, Part 2

[5] He Tao, Applications Analysis of Surface Water Source Heat Pump System, National HVAC Symposium (2004)

[6] Chen Xiao, Application of water source heat pump system utilizing the lake, National HVAC Symposium (2004)

[7] Sun Zongyu, Du Guofu, An analysis of current situation and system simulation of surface water heat pump system, Building energy conservation and green building

[8] Yang Peng, Based on TRNSYS software building ground source heat pump system model and application, Degree paper

[9] Jiang Shiqiang, Hybrid ground source heat pump runs intermittently and economic in hot summer and cold winter, Degree paper 